\title{
Semispinalis Capitis
}

National Cancer Institute

\section{Source}

National Cancer Institute. Semispinalis Capitis. NCI Thesaurus. Code C52989.

One of the transversospinales group of muscles, located in the superior portion of the neck and covering the semispinalis cervicis. It takes its origin from the medial tissue between the superior and inferior nuchal lines of the occipital bone, and inserts into the superior articular processes of the lower four cervical vertebrae and the tips of the transverse processes of the upper six or seven thoracic vertebrae; it functions to extend the neck. 